\title{
Carcinoide intestinal múltiple. Análisis de un caso y revisión de la literatura
}

\author{
Jean Michel Butte $B^{1}$, Cyntia Escobar $F^{2}$, \\ Andrés 0 'Brien $S^{3}$, Alvaro Zúñiga $D^{1}$. \\ Multiple carcinoid tumors of the \\ small bowel. Report of one case
}

Small bowel carcinoid tumors are more common in the distal ileum and they are multiple in $30 \%$ of cases. The most common clinical manifestation is abdominal pain and the treatment of choice is surgical excision. We report a 63 years old female consulting for abdominal pain. An intestinal transit by computed axial tomography revealed multiple images compatible with small bowel carcinoid tumors. She had a surgical excision of the involved intestinal segment and the pathological study confirmed the imaging diagnosis. In the follow up, this patients has been asymptomatic (Rev Méd Chile 2006; 134: 1306-309).

(Key words: Abdominal pain; Carcinoid tumors; Tomography, X-ray)

Recibido el 24 de septiembre, 2005. Aceptado el 26 de abril, 2006.

${ }^{1}$ Departamentos de Cirugía Digestiva, División de Cirugía, ${ }^{2}$ Anatomía Patológica y ${ }^{3}$ Radiología, Facultad de Medicina, Pontificia Universidad Católica de Chile.

L os tumores de intestino delgado son poco frecuentes ${ }^{1}$ y corresponden a $1 \%$ a $2 \%$ de todas las neoplasias del tracto gastrointestinal ${ }^{2,3}$. Generalmente son primarios, aun cuando también han sido descritos como consecuencia de una enfermedad crónica ${ }^{4,5}$. De este grupo, aproximadamente $50 \%$ son adenocarcinomas y $6 \%$ a $35 \%$ son carcinoides 6,7 .

Los carcinoides del intestino delgado se ubican fundamentalmente en el íleon distal y en 30\% son múltiples, no obstante, por su escasa frecuencia, lo anterior es muy difícil de confirmar en la población general ${ }^{8}$. Habitualmente, se presentan en la sexta década de la vida, con dolor abdominal u obstrucción intestinal ${ }^{9}$. Sin embargo, la mayoría de los enfermos no tiene un cuadro característico y pasan muchos años antes del diagnóstico ${ }^{10}$.

El tratamiento, generalmente, es quirúrgico, aun frente a la presencia de metástasis, que la

Correspondencia a: Dr. Alvaro Zúñiga Díaz. Departamento de Cirugía Digestiva, Facultad de Medicina, Pontificia Universidad Católica de Chile. Marcoleta 367, Santiago, Chile. Fono: 56-2-3543870. Fax: 56-2-6329620. E-mail: azuniga@med.puc.cl mayoría de las veces son hepáticas y que tienen un lento crecimiento ${ }^{9}$.

El objetivo de este trabajo es presentar un caso de carcinoides múltiple del intestino delgado y discutir aspectos de esta infrecuente patología.

\section{CASO CLÍNICO}

Paciente de sexo femenino, de 63 años, domiciliada en Santiago. Tiene antecedentes de una resección de un tumor del maléolo peroneo derecho en la infancia, colecistectomía y apendicectomía clásica. Ingresó el 10/6/2005 al Hospital Clínico de la Universidad Católica por un cuadro de 2 días de evolución, caracterizado por dolor abdominal difuso de tipo cólico, distensión abdominal, náuseas y vómitos, y deposiciones diarreicas en aproximadamente 6 veces, luego de una transgresión alimentaria. Durante la evaluación inicial estaba deshidratada, pulso $62 \mathrm{x}^{\prime}$, presión arterial $120 / 80 \mathrm{mmHg}$ y temperatura $36,2^{\circ} \mathrm{C}$. El abdomen se encontraba distendido, sensible en forma difusa, con bazuqueo, sin signos de irritación perito- 
neal y con los ruidos intestinales aumentados en cantidad y tono. Hemograma: hematocrito $47,1 \%$, leucocitos $9.500 \mathrm{x} \mathrm{mm}^{3}$, sin desviación a izquierda y velocidad de eritrosedimentación $21 \mathrm{~mm} / \mathrm{hr}$; proteína $C$ reactiva $0,7 \mathrm{mg} / \mathrm{dl}$ y perfil bioquímico normal. Además, se realizó una tomografía computada de abdomen y pelvis que mostró dilatación de asas a nivel del intestino delgado, con una zona de transición en el íleon medio, donde existía un nódulo intraluminal hiperdenso de 7 $\mathrm{mm}$, que producía una obstrucción intestinal parcial; se asociaba a una adenopatía de aspecto estrellado de $1,3 \mathrm{~cm}$ y a una masa mesentérica hiperdensa de 4 x 2,6 cm. El hígado presentaba 3 lesiones focales inespecíficas en el segmento VI. Fue evaluada por el equipo de cirugía digestiva y se decidió tratar el cuadro clínico en forma médica y completar el estudio de laboratorio e imágenes. La paciente evolucionó en forma satisfactoria, con eliminación de gases y deposiciones a partir del segundo día. El 14/6/05 se realizó una enteroclisis por tomografía computada de abdomen y pelvis, que mostró múltiples lesiones nodulares e hipervasculares en la pared intestinal del yeyunoíleon; estas lesiones abarcaban un segmento de aproximadamente $75 \mathrm{~cm}$. La más distal se encontraba a $35 \mathrm{~cm}$ de la válvula ileocecal, y eran compatibles con la presencia de múltiples carcinoides intestinales. Además, se visualizaron múltiples adenopatías de aspecto estrellado en el mesenterio (Figura 1). Se determinó los niveles del ácido 5-hidroxiindolacético en orina de $24 \mathrm{~h}$, que fue de $47,9 \mu \mathrm{mol} /$ $24 \mathrm{~h}$ (VN hasta 34). La paciente fue intervenida quirúrgicamente el 17/6/2005, encontrándose durante la cirugía múltiples lesiones de aspecto tumoral, algunas con compromiso seroso, en un segmento de 1 metro de yeyunoíleon, la más distal a $40 \mathrm{~cm}$ de la válvula ileocecal. Además, presentaba múltiples adenopatías en el mesenterio, la mayor de $4 \mathrm{~cm}$ y con signos de necrosis. No había metástasis hepáticas ni peritoneales. Se realizó una resección intestinal del segmento comprometido y una anastomosis laterolateral. La paciente tuvo una evolución posoperatoria satisfactoria y fue dada de alta al séptimo día de la cirugía, sin inconvenientes. La biopsia de la pieza quirúrgica confirmó la presencia de múltiples carcinoides bien diferenciados (entre 0,3 y 1,8 cm) (Figura 2A, 2B). En tres de ellos, se observó compromiso de la serosa. Los márgenes fueron

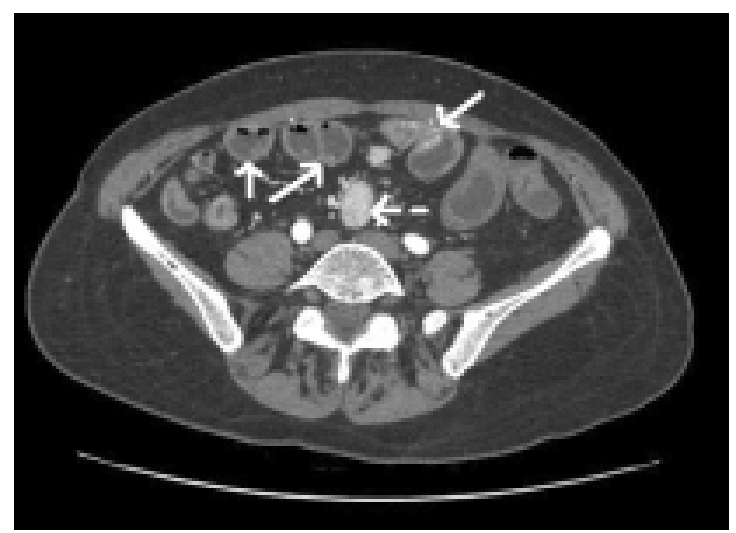

FIgURA 1. Enteroclisis por tomografía computada de abdomen y pelvis: Se observan múltiples lesiones hipervasculares en el íleon proximal (flecha blanca continua), asociado a adenopatías mesentéricas, una de las cuales tiene aspecto espiculado (flecha blanca discontinua).

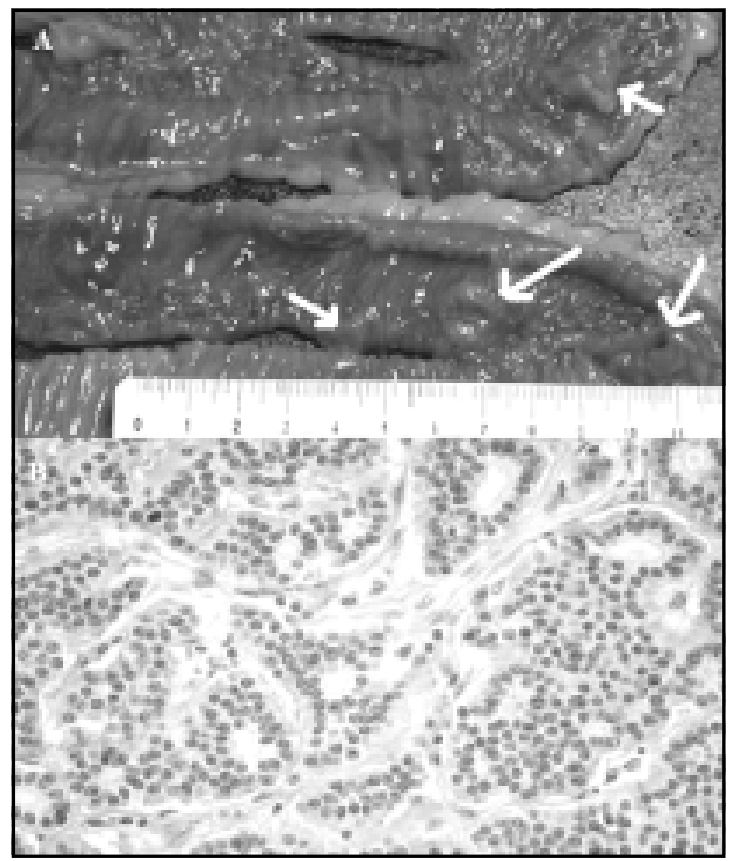

Figura 2. Estudio anatomopatológico: A) Pieza quirúrgica: Se observan múltiples lesiones tumorales, ligeramente solevantadas, aparentemente submucosas, de 0,3 a 1,8 cm de diámetro aproximadamente. B) Tinción de hematoxilina-eosina (aumento 40x). Tumor epitelial, de aspecto organoide, compuesto por células pequeñas de citoplasma eosinófilo claro, núcleos ovoides, de cromatina granular fina y escaso pleomorfismo, dispuestas en pequeños nidos y trabéculas, separados por finas bandas de tejido fibroconjuntivo, compatible con carcinoide intestinal. 
negativos. Un linfonodo, de tres resecados, estaba comprometido por el tumor. Además, se encontraron tres metástasis nodulares en el mesenterio de 4; 1,2 y $0,2 \mathrm{~cm}$. La paciente se encontraba asintomática quince meses después de su operación.

\section{DISCUSIÓN}

Los tumores que actualmente conocemos como carcinoides, fueron descritos por Lubarsch en 1888, al encontrar, en dos autopsias, múltiples lesiones tumorales en el íleon distal ${ }^{11}$. Posteriormente, Oberndorfer ${ }^{12}$, en 1907, describió neoplasias similares, que tenían una conducta menos agresiva que los adenocarcinomas y los denominó carcinoides. Durante muchos años, este término fue utilizado para describir diferentes neoplasias o hiperplasias endocrinas, relacionadas fundamentalmente con la ubicación anatómica, las características morfológicas y un posible potencial biológico común ${ }^{13}$. Actualmente, se sabe que las características clínicas y el pronóstico dependen del origen embrionario del tumor, existiendo diferencias entre aquellos, que derivan del intestino anterior, medio y posterior, tal como lo señalaron Williams y Sobin ${ }^{14}$.

Los carcinoides del yeyuno, íleon, apéndice, colon derecho y ascendente, tienen una génesis común en el intestino medio. Se originan, en la mayoría de los casos, de las células endocrinas del epitelio intestinal, generalmente son bien diferenciados, se asocian a metástasis linfonodales, producen principalmente serotonina $\mathrm{y}$, por esta razón, pueden presentar síndrome carcinoide. Los carcinoides originados en el estómago (intestino anterior) secretan 5 hidroxitriptofano e histamina, por lo que pueden manifestar un síndrome carcinoide atípico. Los carcinoides del colon descendente, sigmoides y recto, no secretan mediadores que produzcan síndrome carcinoide ${ }^{15}$.

Aun cuando la mayoría de los carcinoides se ubican en el íleon distal y en 30\% son múltiples ${ }^{9}$, no existe un consenso sobre la conducta y el tratamiento de ellos, porque no existen muchos casos publicados en la literatura mundial. Yantiss y cols ${ }^{16}$, recientemente, compararon 45 pacientes que presentaron carcinoides intestinales únicos con 18 pacientes que presentaron carcinoides intestinales múltiples, observando que en este último grupo, los tumores se presentaban a una menor edad, había una mayor proporción de mujeres y el síndrome carcinoide era más frecuente. El estudio anatomopatológico de estos tumores múltiples, también mostró que presentan invasión venosa en forma más frecuente y que se encuentran en una etapa más avanzada que los tumores únicos. La enferma aquí descrita y, a diferencia de lo antes señalado, tiene una edad similar al promedio del grupo de tumores únicos, no ha presentado síndrome carcinoide y el tumor no presenta invasión venosa.

El diagnóstico de los carcinoides intestinales es difícil y, en la mitad de los enfermos es incidental $^{17}$, asociado a una patología intercurrente como obstrucción intestinal. El estudio imagenológico con tomografía computada de abdomen y pelvis es fundamental para establecer el diagnóstico previo a la cirugía de la lesión intestinal, de los ganglios linfáticos comprometidos y de las posibles metástasis hepáticas ${ }^{18}$. Es importante considerar que un tercio de ellos son múltiples, por lo que el estudio dinámico del intestino es de mucha utilidad, tal como lo fue la enteroclisis por tomografía computada en esta paciente. Este examen tiene una mayor sensibilidad y especificidad que la tomografía computada convencional, permitiendo la detección de lesiones de hasta 3 $\mathrm{mm}^{19,20}$. Para practicar este examen, se administran 2 litros de agua a través de una sonda nasoenteral ubicada en el ángulo de Treitz y medio de contraste endovenoso. El estudio de imágenes se puede complementar midiendo los niveles del ácido 5-hidroxiindolacético en orina de $24 \mathrm{~h}$. Un aumento de los niveles se asocia a $100 \%$ de especificidad y a $73 \%$ de sensibilidad en el diagnóstico de un tumor carcinoide productor de serotonina ${ }^{18}$, como también ocurrió en esta paciente. Sin embargo, actualmente, el examen más útil para la detección de carcinoides es la cintigrafía con Indio ${ }^{111}$, que permite determinar tumores que tienen receptores de somatostatina ${ }^{18}$. Ochenta y siete por ciento de los carcinoides presentan receptores para somatostatina y este examen tiene una sensibilidad de $75 \%$ y una especificidad de $100 \%$. Nosotros preferimos reservar este estudio para evaluar durante el seguimiento de la paciente la posibilidad de recidiva tumoral, porque los estudios imagenológicos utilizados nos permitieron identificar y resecar la enfermedad macroscópica y porque no mostraban enfermedad en otros sitios. 
En estos pacientes, en que no hay evidencias de enfermedad metastásica, la quimioterapia adyuvante no ha sido validada ${ }^{18}$.

Yantiss y $\mathrm{col}^{16}$, al evaluar la sobrevida, también observaron que los pacientes con tumores múltiples tenían un menor tiempo libre de enfermedad y una menor sobrevida que los pacientes con tumores únicos. Es lógico pensar que esta paciente, que presentaba tumores microscópicos en el intestino resecado, también podría tener lesiones similares en el intestino remanente, por

\section{REFERENCIAS}

1. Talamonti M, Goetz L, Rao S, Joel R. Primary cancers of the small bowel. Analysis of prognostic factors and results of surgical management. Arch Surg 2002; 1237: 564-71.

2. Zownger M. Primary neoplasm of the small intestine. Am Surg 1986; 151: 654-58.

3. Ashley S, Wells S. Tumors of the small intestine. Semin Oncol 1988; 15: 116-28.

4. Butte JM, Dagnino B, Zuñiga DA, Méndez G, Duarte I, Quintana C. Adenocarcinoma de intestino delgado asociado a enfermedad de Crohn. Rev Chil Cir 2003; 55: 509-12.

5. MarchetTI F, Fazio V, Ozuner G. Adenocarcinoma arising from strictureplasty site in Crohn's disease. A case report. Dis Colon Rectum 1996; 39: 1315-21.

6. Cunnnigham J, Aleali R, Aleal M, Brower S, Aufses A. Malignant small bowel neoplasm: histopathologic determinants of recurrence and survival. Ann Surg 1997; 225: 300-6.

7. OJha A, Zacherl J, Scheuba C, Jakesz R, Wenzi E. Primary small bowel malignancies: single-center results of three decades. J Clin Gastroenterol 2000; 30: 289-93.

8. Memon M, NeLSON H. Gastrointestinal carcinoid tumors: current management strategies. Dis Colon Rectum 1997; 40: 1101-18.

9. KULKE M, MAYER R. Carcinoid tumors. NEngl J Med 1999; 340: 858-68.

10. Moertel C, Sauer W, Dockerty M, Baggenstoss A. Life history of the carcinoid tumor of the small intestine. Cancer 1961; 14: 901-12. lo que el seguimiento debe ser estrecho y programado, fundamentalmente con imágenes y con cintigrafía de receptores de somatostatina ${ }^{18}$, con el fin de detectar una posible progresión de la enfermedad lo más precoz posible.

Finalmente, es necesario tener presente que, en general, los carcinoides del intestino delgado, aunque se presenten con metástasis, tienen una lenta progresión $\mathrm{y}$, por lo tanto, se justifica practicar resecciones paliativas que disminuyan los síntomas y mejoren la calidad de vida ${ }^{20}$.

11. Lubarsch O. Ueber den primaren Krebs des ileum, nebst Bemerkungen über das Gleichzeitige Vorkommen von Krebs und Tuberkolose. Virchows Arch 1888; 111: 280-317.

12. OberNDORFER S. Karzinoide Tumoren des Dünndarms. Frankf Z Pathol 1907; 1: 425-9.

13. Burke A, Thomas R, Eisayed A, Sobin L. Carcinoids of the jejunum and ileum: an immunohistochemical and clinicopathologic study of 167 cases. Cancer 1997; 79: 1086-93.

14. Wiшums E, Sobin M. The classification of carcinoid tumours. Lancet 1963; 1: 238-9.

15. Sutton R, Doran H, Wimams E, Vora J, Vinjamuri S, EvanS J ET AL. Surgery for midgut carcinoid. Endocrine-Related Cancer 2003; 10: 469-81.

16. Yantiss R, Odze R, Farraye F, Rosenberg A. Solitary versus multiple carcinoid tumors of the ileum. A clinical and pathologic review of 68 cases. Am J Surg Pathol 2003; 27: 811-7.

17. Shebani K, Souba W, Finkelstein D, Stark P, Elgadi $\mathrm{K}$, Tanabe $\mathrm{K}$ et al. Prognosis and survival in patients with gastrointestinal tract carcinoid tumors. Ann Surg 1999; 229: 815-23.

18. Sippel R, CHen H. An update on carcinoid tumors. Probl Gen Surg 2003; 20: 125-33.

19. Boudiaf M, JafF A, Soyer P, Bouhnik Y, Hamzi L, RYMER R. Small-bowel diseases: prospective evaluation of multi-detector row helical CT enteroclysis in 107 consecutive patients. Radiology 2004; 233: 338-44.

20. Boudreaux J, Putty B, Frey D, Woltering E, Lowell A, DALY I ET AL. Surgical treatment of advancedstage carcinoid tumors: lessons learned. Ann Surg 2005; 241: 839-46. 